Please do not remove this page

RMIT

UNIVERSITY

\title{
Colour image enhancement by hybrid approach
}

Xu, Zhengya; Wu, Hong Ren; Yu, Xinghuo

https://researchrepository.rmit.edu.au/esploro/outputs/9921863726101341/filesAndLinks?institution=61RMIT_INST\&index=null

Xu, Z., Wu, H. R., \& Yu, X. (2008). Colour image enhancement by hybrid approach. Proceedings of the Institute of Electrical and Electronics Engineers 10th Workshop on Multimedia Signal Processing, 252-256. https://doi.org/10.1109/MMSP.2008.4665084

Published Version: https://doi.org/10.1109/MMSP.2008.4665084

Repository homepage: https://researchrepository.rmit.edu.au (C) 2008 IEEE

Downloaded On 2023/04/26 13:02:46 +1000

Please do not remove this page 


\title{
Colour Image Enhancement by Hybrid Approach
}

\author{
Zhengya $\mathrm{Xu}^{\# 1}$, Hong Ren $\mathrm{Wu}^{\# 2}$, Xinghuo $\mathrm{Yu}^{\# 3}$ \\ ${ }^{\#}$ School of Electrical and Computer Engineering, Platform Technologies Research Institute, RMIT University \\ VIC 3001, Australia \\ zhengya.xuermit.edu. au \\ 2.henry.wuermit.edu.au \\ $\mathrm{x} \cdot$ yuermit.deu.au
}

\begin{abstract}
This paper introduces a novel image enhancement methodology driven by both global and local processes. This methodology, based on the parameters controlled hybrid approach which integrates the advantages of point operations with local enhancement techniques, can enhance, simultaneously, the overall contrast and the sharpness of an image and increase, especially, the visibility of specified portions or aspects of the image. The methodology was compared with other classical image enhancement techniques, such as linear contrast stretching and histogram equalization. Results obtained in terms of subjective evaluation have shown the superiority of the proposed approach.
\end{abstract}

\section{INTRODUCTION}

Image enhancement, which transforms digital images to enhance the visual information within, is a primary operation for almost all vision and image processing tasks in several areas such as computer vision, biomedical image analysis, forensic video/image analysis, remote sensing and fault detection.

First of all, the basic strategies are briefly reviewed for image enhancement. Point-operation-based image enhancement includes contrast stretching and histogram modelling. They are zero memory operations that remap a given input gray-level into an output gray-level, according to a global transformation [2], [4], [9]. These methods have the disadvantage of treating the image globally, not being able to differentiate between several areas of the image that may require different levels of contrast enhancement. Other classes of methods for image enhancement are spatial operations and pseudo-colouring. Spatial operations may suffer from enhancing excessively the noise in the image or conversely smoothing the image in areas which need sharp details [3] and these operations are also known to be time consuming. Pseudo-colouring methods artificially map the gray-scale image based on colour mapping, with the disadvantage that extensive interactive trials are required to determine an acceptable mapping scheme [2].

Local enhancement methods have been developed based on the gray-level distribution in the neighbourhood of every pixel in a given image. One typical example of local enhancement methods is the adaptive histogram equalization (AHE), which has shown good results in medical imaging applications [5]. However, AHE uses an enhancement kernel that is quite computationally expensive. Moreover, AHE may yield unsatisfactory outputs, e.g., images with noise artefacts and falsely enhanced shadows.
Recently, in automatic image enhancement, evolutionary algorithms (e.g., EVOLEHA [1]) have been applied [6]-[8]. The authors apply a global contrast enhancement technique using genetic programming (GP) [10] to adapt the colour map in the image as to fit the demands of the human interpreter. However this sort of enhancement techniques is only suitable for routine application and the computational cost is high [1].

Based on above analysis, the proposed image enhancement methodology is a fast adjustable hybrid approach controlled by a number of parameters in order to take the full advantages of point operations and local information driven enhancement techniques, and to avoid their shortcomings. The organization of this paper is as follows. Section II addresses the principles of the proposed image enhancement technique. Experimental results are presented in Section III. Final conclusions are drawn in Section IV.

\section{PRINCIPLE OF THE PROPOSED METHODOLOGY}

The brightness scale transformation is used when a human observer views a colour image and a transformed image might be more easily interpreted if the contrast is enhanced. For this type of enhancement, usually the histogram equalization technique is used to create an image with equally distributed brightness levels over the entire brightness scale. Though histogram equalization applies a transformation that yields a close-to-uniform histogram for the relative frequency of the gray-levels in an image, histogram equalization only enhances contrast for brightness value close to histogram maxima, and decreases the contrast near histogram minima. If the image analyst is interested in certain parts of an image and the brightness of the parts of the image is not close the histogram maxima, or even near the histogram minima, histogram equalization is helpless in contrast enhancement. Linear brightness stretching is only effective for an image where the histogram is narrow [9].

In order to meet the above particular practical demands which are stringent requirements for biomedical image analysis, forensic image/video analysis and remote sensing, a new colour image enhancement methodology is proposed as follows. The proposed new enhancement technique is driven by both global and local processes to achieve, not only effective improvement of overall contrast but also the significant enhancement of details in identified areas of interest, of a colour image. The proposed methodology must also employ a much less time-consuming enhancement mechanism. Therefore, the intention of the proposed method 
is to find a monotonic pixel brightness transformation $q=T(p)$ for an colour image such that the desired output histogram can not only meet specific requirements but also as uniform as possible over the whole output brightness scale [9] to fill the full range of brightness values.

First of all, if needed, the colour image is transformed from RGB colour space or other colour space to YUV/YIQ colour space. Suppose that an input histogram is denoted as $h(p)$ and the input brightness value is $\left[p_{0}, p_{k}\right]$, we denote $H=\sum_{i=0}^{k} h\left(p_{i}\right)$.

Compared with the original image, an enhanced image with good contrast will have a higher intensity of the edges [7]. Since we are interested only in the edges intensity without regard to their orientation, a linear differential operator called the Laplacian, $\nabla^{2} g(x, y)=\frac{\partial^{2} g(x, y)}{\partial x^{2}}+\frac{\partial^{2} g(x, y)}{\partial y^{2}}$ may be used.

If $L$ is an output brightness value of a pixel with original brightness of $p_{i}$ in an image after the image processed with a Laplace operator $\nabla^{2}$, and the $h_{v}\left(p_{i}\right)$ is sum of $L$ of all pixels with the brightness of $p_{i}, h_{v}\left(p_{i}\right)$ can be treated as a special probability density function depended on the local feature of every pixel and the frequency of the brightness value of the pixels. Define $H_{1}$ as,

$$
H_{1}=w \sum_{i=k 10}^{k 1} h\left(p_{i}\right)=w \sum_{i=k 10}^{k 1} h_{k}\left(p_{i}\right)
$$

where $k l$ and $k 10$ are in the range of $[0, k] ; h_{k}\left(p_{i}\right)=h\left(p_{i}\right)$ if $p_{i}$ is in the range of $\left(p_{k 10}, p_{k 1}\right)$, otherwise $h_{k}\left(p_{i}\right)=0 ; w$ is a parameter with the default value of 2 . After normalization by the input brightness range, define $H_{2}$ as,

$$
H_{2}=\frac{v}{p_{k}-p_{0}} \sum_{i=0}^{k} h_{v}\left(p_{i}\right)
$$

where $v$ is a parameter with the default value of 2 .

$$
\text { If } c=\frac{H}{H+H_{1}+H_{2}} \text {, a new distribution function is defined }
$$

as

$$
\sum_{i=p_{0}}^{p} h_{0}(i)=c\left(\sum_{i-p_{0}}^{p} h(i)+w \sum_{i-P_{0}}^{p} h_{k}(i)+\frac{v}{p_{k}-p_{0}} \sum_{i-P_{0}}^{p} h_{v}(i)\right)
$$

where $p$ is in $\left[p_{0}, p_{k}\right]$.

Assuming that $N$ and $W$ are the height and the width of an image, respectively, and the output brightness scale is $\left[q_{0}, q_{k}\right]$, the desired output histogram can be obtained by the following its corresponding continuous probability density equation,

$$
W N \int_{q_{0}}^{l} \frac{1}{q_{k}-q_{0}} d s=\int_{p_{0}}^{p} h_{0}(s) d s
$$

The left side of Equation (4) is the corresponding probability uniform distribution function. The desired pixel brightness histogram transformation $T$ can further be derived as

$$
q=T(p)=\frac{q_{k}-q_{0}}{W N} \int_{p_{0}}^{p} h_{0}(s) d s+q_{0}
$$

Now we try to obtain a quantisation of the number of brightness-levels present in the output image by (5). The discrete approximation of the continuous pixel brightness transformation from Equation (5) is

$$
q=T(p)=\frac{q_{k}-q_{0}}{W N} \sum_{i=P_{0}}^{p} h_{0}(i)+q_{0}
$$

After derivation and discrete approximation of the above Equation (6), we can obtain the following equations,

$$
\begin{aligned}
& \Delta q=\frac{q_{k}-q_{0}}{W N} h_{0}(p) \\
& =\frac{q_{k}-q_{0}}{W N}\left(h(p)+w h_{k}(p)+\frac{v}{p_{k}-p_{0}} h_{v}(p)\right)
\end{aligned}
$$

On the right side of Equation (7), the second term is used to enhance contrast for a specified range $\left(p_{k 10}, p_{k l}\right] ; w h_{k}(p)=0$, if $p_{i}$ is not in the range of $\left(p_{k 10}, p_{k l}\right]$; the third term, basically as the first term (input histogram of the image), is dependent on the image structure, though the parameter $v$ could be adjusted. In most cases the $v$ is fixed as 1 . Through (7), it can be clearly seen how the output interval value between adjacent two brightness values is produced one by one and how the parameters make contribution to every output bright level for contrast enhancement. The default values of these parameters are: $k 10=0, k l=30$ and $w=2$. The parameters could be adjusted by an image interpreter to meet his or her specific requirements. For many cases, the proposed approach with the default values of the parameters works well without user intervention.

When enhancing the contrast of the dark area of an image whose histogram spans a broad range of the display scale, the bright area of the image will be out of the display range as a result of linear stretching. Therefore, a hard-limit is needed to map the output image pixel values back into the display range [9]. However, the simple hard-limiting method is only suitable for an output image with only a few pixels whose brightness values are outside $\left[q_{0}, q_{k}\right]$.

In order to avoid or greatly reduce the brightness range of the output image, a rescaling process is employed using parameter $t$, which is to be added in the methodology, to limit the maxima of $\nabla q$ within $t$, and to smooth the enhancement contrast over the full brightness scale. The default value of $t$ is 4. The rescaling process, also further relieves undesired property of the applied equalisation histogram technique reducing contract near histogram minima. Since in some cases the last $\nabla q$ of the transformation by Equation (7) is very large, linear contrast stretch transformation is also applied in the proposed approach to ensure full use of the output brightness scale.

Finally, after the brightness contrast enhancement, the output colour image is transformed back to RGB colour space or other original colour space.

\section{EXPERIMENTS}

In order to evaluate our method, the proposed method is compared with two classical enhancement methods: linear contrast stretching and histogram equalization [2] using eight test images [11]. Without losing generality, the test images include well-known typical test images such as, Boats, Girl, 
Gold hill, Fruits, Pen, Cable car, Airplane and Meat with an image resolution of $500 \times 362$ or $512 \times 512$ or $787 \times 576$ or $720 \times 576$ or $768 \times 512$ pixels.

It is observed from the experimental results shown in Figs. 1 and 2 that the proposed enhancement algorithm can effectively enhance the overall contrast and the sharpness of the test images. A lot of details that could not be seen in the original images have been clearly revealed. From the output images, although these images were enhanced by histogram equalization, the histogram equalization also over-enhanced some part of the image and highlighted block noise artefacts

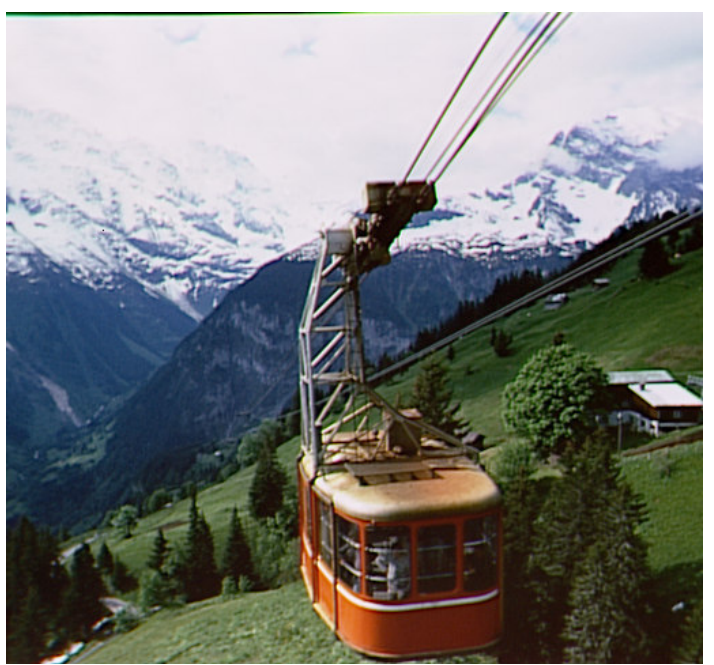

a)

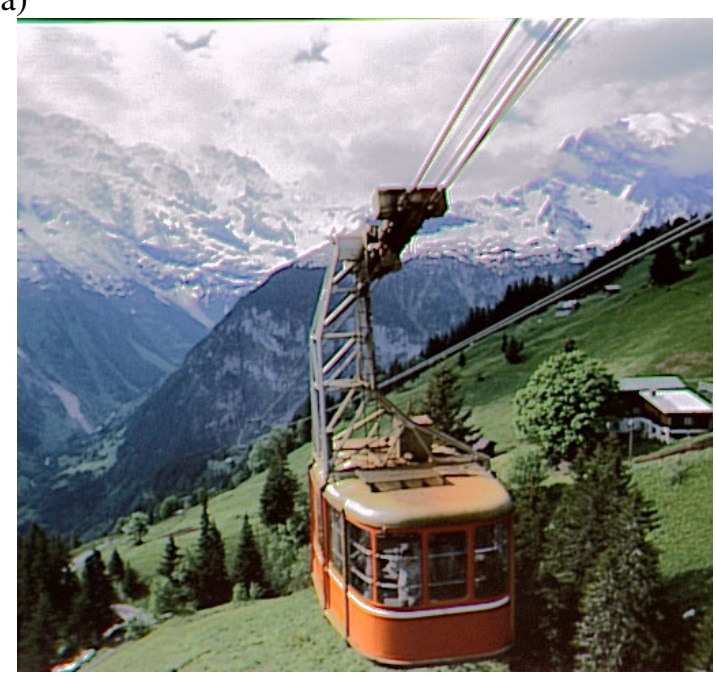

c) caused by image compression (see Fig. 2), as the pixels representing block noise artefacts have brightness value close to histogram maxima. For revealing the dark details in images with a broad low histogram, the performance of the proposed approach is much better than the other two methods. The details in the dark areas of the images "Cable car" are much more visible than those in the original image. The linear stretching failed to make significant enhancement for these two images.

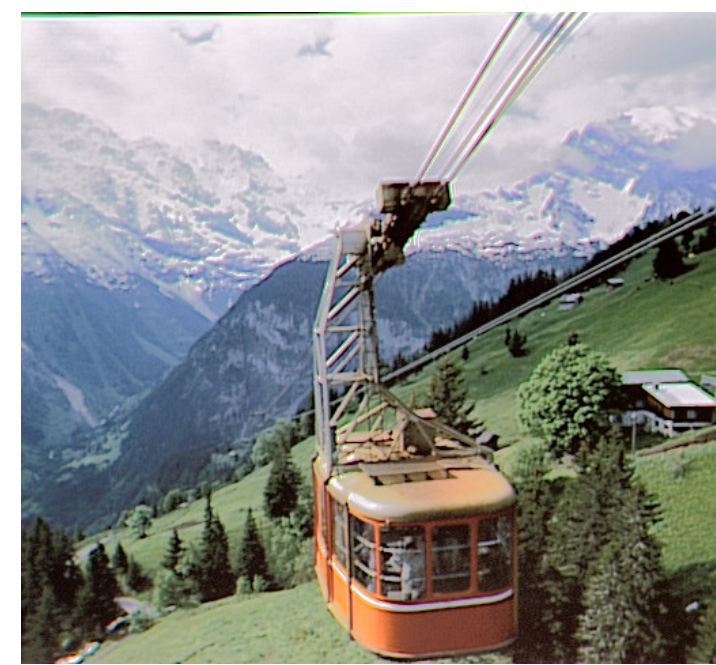

b)

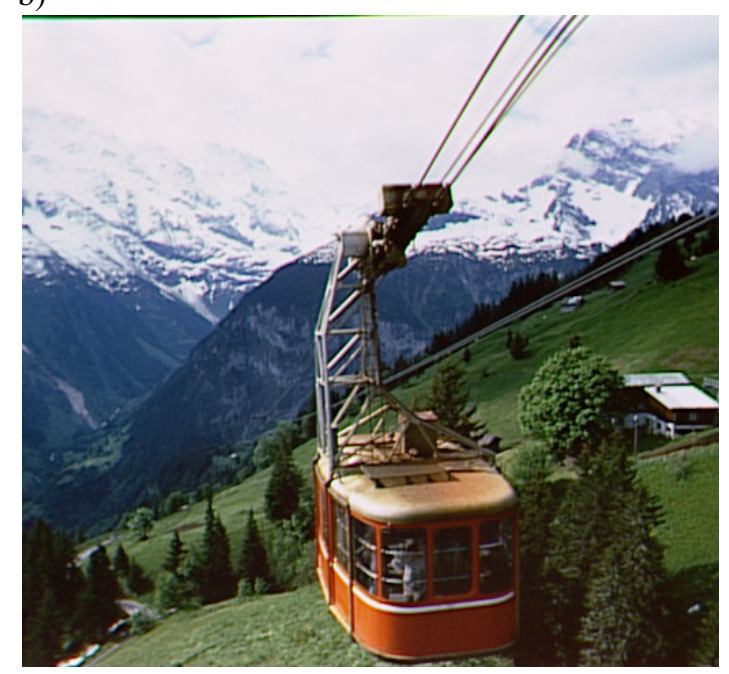

d)

Fig. 1. The enhancement results for test image "Cable car", a) Original image, b) output of the proposed approach, c) output of histogram equalisation, d) output of linear stretching 

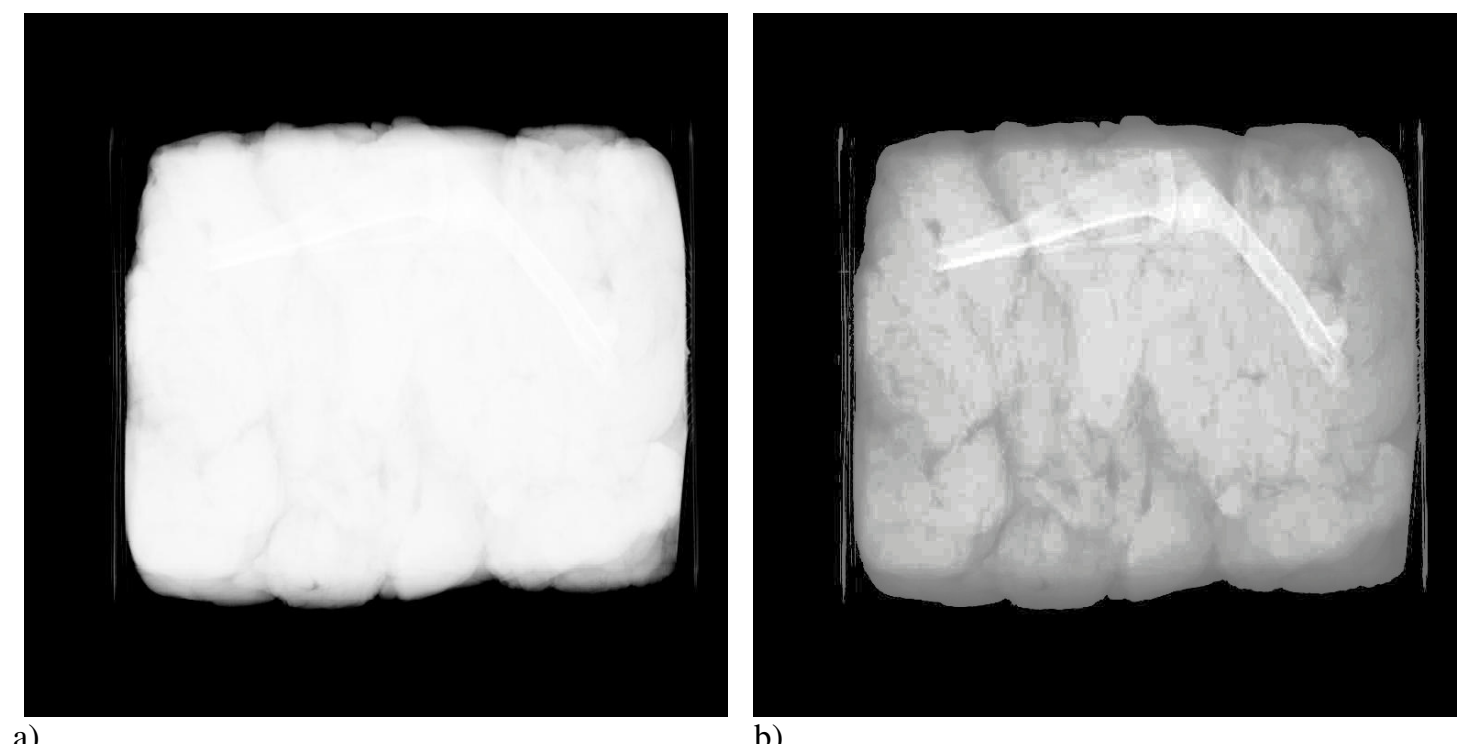

a)

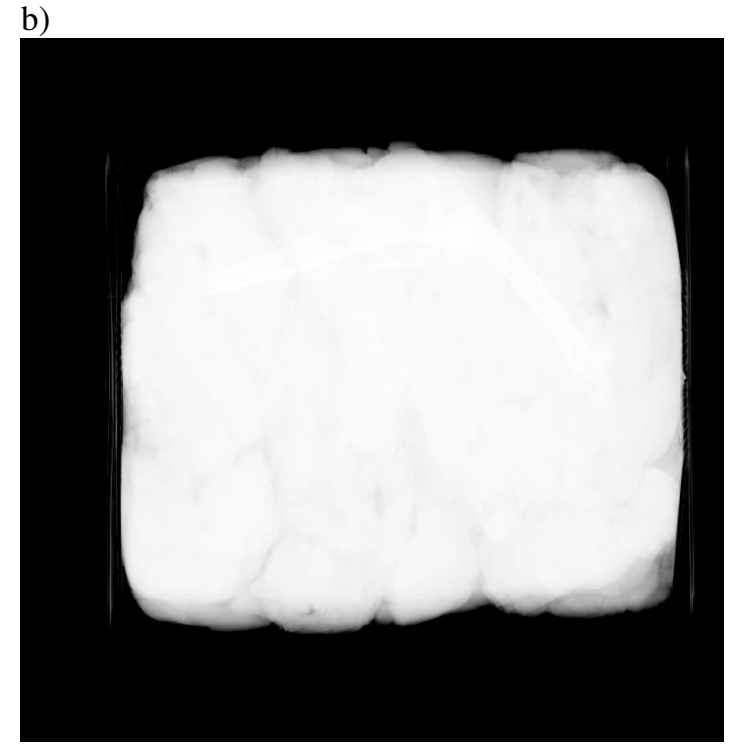

c)

d)

Fig. 2. The enhancement results for test image "Meat", a) Original image, b) output of the proposed approach, c) output of histogram equalisation, d) output of linear stretching

Table I Subjective Evaluation Result

\begin{tabular}{|l|l|l|l|l|l|l|l|l|l|l|l|l|}
\hline Methods & \multicolumn{4}{|l|}{ Linear Stretching } & \multicolumn{4}{l|}{$\begin{array}{l}\text { Histogram } \\
\text { Equalization }\end{array}$} & \multicolumn{4}{l|}{$\begin{array}{l}\text { The proposed } \\
\text { Approach }\end{array}$} \\
\hline Rank & 1 & 2 & 3 & Average & 1 & 2 & 3 & Average & 1 & 2 & 3 & Average \\
\hline Cable & 0 & 2 & 4 & 2.6 & 0 & 4 & 2 & 2.3 & 6 & 0 & 0 & 1.0 \\
\hline Boats & 1 & 3 & 2 & 2.2 & 1 & 1 & 4 & 2.5 & 4 & 2 & 0 & 1.3 \\
\hline Girl & 1 & 2 & 3 & 2.3 & 1 & 2 & 3 & 2.3 & 4 & 2 & 0 & 1.3 \\
\hline Gold hill & 0 & 2 & 4 & 2.6 & 0 & 4 & 2 & 2.3 & 6 & 0 & 0 & 1.0 \\
\hline Fruits & 0 & 2 & 4 & 2.6 & 0 & 4 & 2 & 2.3 & 6 & 0 & 0 & 1.0 \\
\hline Meat & 0 & 1 & 5 & 2.8 & 1 & 4 & 1 & 2.0 & 5 & 1 & 0 & 1.2 \\
\hline Airplane & 2 & 3 & 1 & 1.8 & 0 & 1 & 5 & 2.8 & 4 & 2 & 0 & 1.3 \\
\hline Pens & 2 & 2 & 2 & 2.0 & 2 & 2 & 2 & 2.0 & 4 & 2 & 0 & 1.3 \\
\hline Total & & & & 2.6 & & & & 2.4 & & & & 1.2 \\
\hline
\end{tabular}


In order to evaluate the performances of the image enhancement techniques, six human viewers (two of whom were image processing experts) evaluated subjectively all the test images produced by the three methods. Each image had to be ranked by giving a score ranging 1-3 and the best score is 1 . The subjective criterion was natural brightness/contrast for the enhanced images. Results are given in Table I. The proposed approach ranks best for each image. Thus, the proposed algorithm holds the potential for many applications, such as real-time medical image and forensic video/image enhancements.

\section{CONCLUSION}

In this paper, a new hybrid approach for colour image enhancement is proposed. Through experiments conducted using a wide range of colour images, the proposed approach has demonstrated superior performance to that of the classical point operations (linea contrast stretching and histogram equalization), in terms of effectiveness of colour image contrast enhancement. The proposed hybrid approach applies techniques of histogram equalization, linear stretching and local feature based enhancement, with significant modifications. Global contrast enhancement was also achieved by a specified function based on intensity of edges to better adapt to the local features in colour images. The proposed methodology introduces the parameters, which are employed to increase the visibility of specified portions or aspects of the image.
The proposed methodology is very useful for better enhancing specific categories of images, such as biomedical images, forensic image/video and satellite images.

\section{REFERENCES}

[1] Cristian Munteanu and Agostinho Rosa, "Gray-Scale Image Enhancement as an Automatic Process Driven by Evolution," IEEE Transactions on Systems, Man, and Cybernetics-part b: Cybernetics, vol. 34, no. 2, April 2004.

[2] W. K. Pratt, Digital Image Processing. New York: Wiley, 2000.

[3] G. Ramponi, N. Strobel, S. K. Mitra, and T.-H. Yu, "Nonlinea un-sharp masking methods for image contrast enhancement," J. Electron. Imaging, vol. 5, no. 3, pp. 353-366, 1996.

[4] K. Jain, Fundamentals of Digital Image Processing. Englewood Cliffs, NJ: Prentice-Hall, 1991.

[5] J. B. Zimmerman, S. M. Pizer, E. V. Staab, J. R. Pery, W. McCartney, and B. Brenton, "An evaluation of the effectiveness of adaptive histogram equalization for contrast enhancement," IEEE Trans. Med. Imag., vol. 7, pp. 304-312, Dec. 1988.

[6] C. Munteanu and V. Lazarescu, "Evolutionary contrast stretching and detail enhancement of satellite images," in Proc. Mendel, B no, Czech Rep., 1999, pp. 94-99.

[7] F. Saitoh, "Image contrast enhancement using Genetic Algorithm," in Proc. IEEE SMC, Tokyo, Japan, 1999, pp. 899-904.

[8] C. Munteanu and A. Rosa, "Evolutionary image enhancement with use behaviour modelling," Appl. Comput. Rev., vol. 9, no. 1, pp. 8-14, 2001.

[9] G. Deng, L. W. Cahill, and G. R. Tobin, "The Study of Logarithmic Image Processing Model and Its Application to Image Enhancement," IEEE Trans. on Image Processing, vol. 4. no. 4, April 1995.

[10] T.Bäck et al., Eds., "Handbook of Evolutionary Computation," Oxford University. Press, London, U.K., 1997.

[11] http://www.hlevkin.com. 